\title{
Binarity and Variable Stars in the Open Cluster NGC 2126
}

\author{
Nareemas Chehlaeh $^{1,2 *}$, David Mkrtichian ${ }^{3}$, Seung-Lee Kim ${ }^{4}$, Patricia Lampens ${ }^{5}$, \\ Siramas Komonjinda ${ }^{1}$, Anatoly Kusakin ${ }^{6}$, Ljudmila Glazunova ${ }^{7,8}$
}

\author{
${ }^{1}$ Department of Physics and Materials Science, Faculty of Science, Chiang Mai University, Thailand \\ ${ }^{2}$ Graduate School, Chiang Mai University, 50200, Chiang Mai, Thailand \\ ${ }^{3}$ National Astronomical Institute of Thailand, 50200, Chiang Mai, Thailand \\ ${ }^{4}$ Korea Astronomy and Space Science Institute, 34055, Daejeon, Korea \\ ${ }^{5}$ Koninklijke Sterrenwacht van België, Ringlaan 3, B-1180, Brussel, Belgium \\ ${ }^{6}$ Fesenkov Astrophysical Institute, Observatory street, 23, 050020, Almaty, Kazakhstan \\ ${ }^{7}$ Astronomical Observatory, Odessa National University, Shevchenko Park, 65014, Odessa, Ukraine \\ ${ }^{8}$ Odessa National Academy of Telecommunications, Kuznechnaya street 1, 65029, Odessa, Ukraine
}

\begin{abstract}
We present the results of an analysis of photometric time-series observations for NGC 2126 acquired at the Thai National Observatory (TNO) in Thailand and the Mount Lemmon Optical Astronomy Observatory (LOAO) in USA during the years 2004, 2013 and 2015. The main purpose is to search for new variable stars and to study the light curves of binary systems as well as the oscillation spectra of pulsating stars. NGC 2126 is an intermediate-age open cluster which has a population of stars inside the $\delta$ Scuti instability strip. Several variable stars are reported including three eclipsing binary stars, one of which is an eclipsing binary star with a pulsating component (V551 Aur). The Wilson-Devinney technique was used to analyze its light curves and to determine a new set of the system's parameters. A frequency analysis of the eclipse-subtracted light curve was also performed. Eclipsing binaries which are members of open clusters are capable of delivering strong constraints on the cluster's properties which are in turn useful for a pulsational analysis of their pulsating components. Therefore, high-resolution, high-quality spectra will be needed to derive accurate component radial velocities of the faint eclipsing binaries which are located in the field of NGC 2126. The new Devasthal Optical Telescope, suitably equipped, could in principle do this.
\end{abstract}

\section{Introduction}

One of the important ways for understanding the history of star formation is the study of Galactic open clusters. Open cluster members have a common formation history (age), metallicity, distance, environment and allow to determine these parameters using theoretical isochrone fitting (e.g. Tapia et al. 2010; Glushkova et al. 2013). Open clusters with an age of 0.3-1 Gyr and a reasonable distance

*e-mail: nareemas.c@gmail.com 
of 1-2 kpc are suitable for studying short-period pulsators, especially $\delta$ Scuti type stars (Frandsen $\&$ Arentoft 1998). The main advantage of studying pulsating stars in clusters is the cluster properties that can be applied to these members. Such properties are generally poorly known for single pulsating stars in the field. Knowledge of these properties may help to better constrain the stellar pulsation models. The advantages of studying binary systems in clusters is that the observed properties of the detached components in the mass-radius-temperature diagrams must match the overall properties of the cluster to which the system belongs. Detached eclipsing binaries with well-determined masses and radii are particularly important. These combinations provide a set of constraints which allow to find more accurate solutions and to study the characteristics of stars and clusters together.

NGC $2126\left(\mathrm{RA}_{2000}=06^{\mathrm{h}} 02^{\mathrm{m}} 55^{\mathrm{s}}, \mathrm{Dec}_{2000}=+49^{\circ} 52^{\prime}\right)$ is an intermediate-age and moderately rich typical galactic cluster with several dozens of members scatters in a region of $5^{\prime}-6^{\prime}$ in the constellation Auriga in the northern sky (Lynga 1995). First observations were made by Cuffey (1943), who presented blue and red photographic observation and estimated the distance to the cluster to be 950 pc, by comparing the observed color-magnitude relations to those of M38. The first CCD photometric observation was published by Gaspar et al. (2003). They calculated the parameters of the cluster and discovered six new variable stars in the field, including V551 Aur which is an eclipsing binary with pulsating component with an orbital period of $\mathrm{P}_{\text {orb }}=1.173 \mathrm{~d}$. They presented some characteristics of the cluster: the distance modulus $m-M=11.0 \pm 0.5 \mathrm{mag}$, the reddening $E(B-V)=0.2 \pm 0.15$ mag, a distance to the cluster of $1.3 \pm 0.6 \mathrm{kpc}$ (assuming $A_{v} \approx 3.1 \times E(B-V)$ ), and an age of 9.0 $<\log _{10}(t)<9.4$. In 2004, we acquired new photometry of the cluster and discovered several new $\delta$ Scuti stars. This result was not published yet as we aimed to obtain new, more extensive photometry. After that, Liu et al. (2009) discovered additional variables stars in a field of about one degree centered on the cluster.

In the present paper, we discuss the analysis of the eclipsing binary system V551 Aur. The observations and data reduction are presented in Section 2. The statistics on detected variable stars in NGC 2126 is briefly discussed in Section 3. The eclipsing binary with pulsating component V551 Aur is presented in Section 4. Section 5 is followed by a discussion of our results and conclusions.

\section{Observations and data reduction}

The CCD images in $V$ band were carried out during 22 nights separated into three observing runs (between 2004 and 2015). The first season was between 24th and 31th March 2004 at the Mount Lemmon Optical Astronomy Observatory Arizona (LOAO), Arizona, using the 1.0-m robotic telescope (Han et al. 2005). The detector was a $2 \mathrm{k} \times 2 \mathrm{k}$ CCD camera. The field of view (FOV) of a CCD image is about $22.2^{\prime} \times 22.2^{\prime}$. A total of 515 images were obtained in the $V$-band, with an exposure time of about 100-300 s. The second run was between 12th January and 9th February 2013 using the 2.4-m Thai National Telescope (TNT). The detector was a $2 \mathrm{k} \times 2 \mathrm{k}$ Apogee U4200 CCD camera. The FOV of the CCD covers about $5.5^{\prime} \times 5.5^{\prime}$ on the sky. In total, 456 images were obtained in the $B$ and $V$ bands, with an exposure time of about $200 \mathrm{~s}$. In the last period, between 16th and 21st March 2015, we used the 0.5-m Corrected Dall-Kirkham PlaneWave CDK24 remote-controlled telescope located at the Thai National Observatory (TNO). The detector was an Apogee Altra U9000 3056×3056 pixel CCD camera. The FOV of the CCD covers about $22.2^{\prime} \times 22.2^{\prime}$. For this observing run, a total of 411 images were obtained in the $V$ filter, with an exposure time of about $100 \mathrm{~s}$.

The CCD frame processing was performed using the standard routines of CCDPROC in the IRAF package (Stetson 1987) to measure the differential magnitude of the stars in the FOV. Table 1 shows the coordinates in the UCAC4 catalog (Zacharias et al. 2012) of the variable, reference and check stars. 


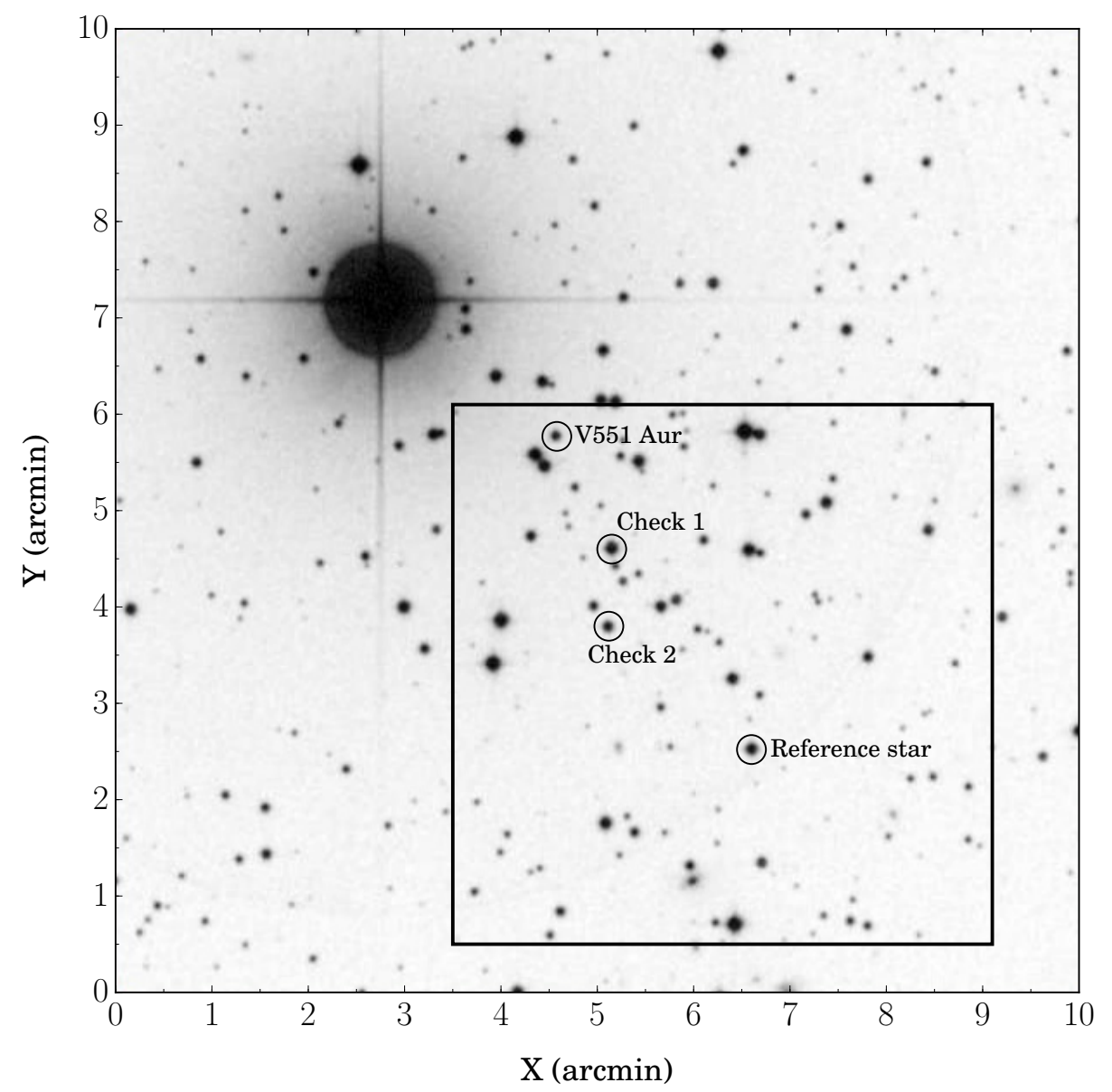

Figure 1: Digitized sky survey image of NGC $2126\left(10^{\prime} \times 10^{\prime}\right.$, north is up, east is to the left)). The black box shows the FOV covered by our observations in 2013.

\section{New Variables}

We performed a Discrete Fourier Transform (DFT) period analysis for all stars in the observed field of view showing any variability. From these period analyses, we distinguished in total eleven eclipsing binaries and pulsating variable stars. Two of them are new $\delta$ type pulsating stars according to their light variation behaviors and their position in the color-magnitude diagram (CMD). The nine others are known variables discovered by Gaspar et al. (2003) and Zhang et al. (2012).

\section{The eclipsing binaries with pulsating component V551 Aur}

Eclipsing binaries with pulsating components are very crucial object for asteroseismology, because they allow for independent determination of physical parameters like the mass, radii, and temperature of the components. The monitoring of eclipse phenomena provides a tool for mode-identification (Mkrtichian et al. 2002) and to compare the results with theoretical models. The study of pulsating stars in binaries gives new constrains for stellar theories and for the understanding of the influences 
Table 1: Coordinates from UCAC4 catalog of the variable, reference, and check stars

\begin{tabular}{cccc}
\hline Name & UCAC4 Catalog & $\alpha(\mathrm{J} 2000)$ & $\delta(\mathrm{J} 2000)$ \\
\hline V551 Aur & $700-043241$ & 060238.044 & +495302.48 \\
Reference star & $700-043189$ & 060226.332 & +495002.35 \\
Check star 1 & $700-043225$ & 060234.704 & +495157.93 \\
Check star 2 & $700-043227$ & 060234.941 & +495112.88 \\
\hline
\end{tabular}

of tidal forces and mass-transfer (Zhang et al. 2014).

V551 Aur is a 1.173-day detached eclipsing binary system with a pulsating component. It was discovered by Gaspar et al. (2003). The system shows steady $\delta$ Scuti-like oscillation outside eclipse, with an amplitude 0.05 mag and a pulsation period of 0.12935 days. There might be a 1:9 resonance between the orbital motion and the pulsation. Gaspar et al. (2003) classified the primary component as an early F-type star. This classification was later confirmed by Liu et al. (2009). Liu et al. (2012) suggested that the primary component of the system is very probably a g-mode pulsator. The aim of current investigations is to find accurate parameters of the binary system, including the orbital and pulsation periods, in order to check the resonance hypothesis.

\subsection{Binary System}

Twenty eclipses from Gaspar et al. (2003), Liu et al. (2012) and our data are recorded in Table 2. The three epochs of primary light minima originating from this research from 2004 and 2013 data along with others compiled from the literature were used to find the new ephemeris equation. By using the least square fitting method, the new linear ephemeris is found to be:

$$
\operatorname{Min.I}(H J D)=2452640.59671+1.1731744(2) E
$$

With the new ephemeris, we obtained the phase diagram as shown in Fig. 2. The small offsets between the $V$ band light curves from the data of the different seasons (2004, 2013, and 2015) were calculated. We obtained the best offset values by using a Fortran program (written by L. Vermeylen, ROB) to shift the respective phased light curves. The program computes the offset values from the minimization of the residuals in the phase diagram and writes out the best values between two data sets. The resulting shifts are 0.038 (for the year 2013) and 0.017 (for the year 2015) mag compared to the 2004 phase diagram as shown in Fig. 2. For one rising branch of the primary minimum, observed in 2015, we had to use a supplementary offset, however.

The light curve was analyzed using the program PHOEBE (Prša \& Zwitter 2005) which is based on the Wilson-Devinney code with Kuruczs atmosphere models (Wilson \& Devinney 1971). The estimation of stellar absorption obtained for the open cluster NGC $2126(E(B-V)=0.20 \pm 0.15$; Gaspar et al. 2003) was used to calculate the temperature of the primary star, $T_{1}$, which is in the range of 7000-7250 K. In the search of an eclipsing binary model, $T_{1}$ was set to $7000 \mathrm{~K}$. The bolometric and monochromatic limb darkening coefficients were taken from van Hamme (1993). We considered either a convective or a radiative flux transport approximation. The best model was found to have a convective envelope for the primary star. Thus the bolometric albedo of the components were set to $A_{1}=A_{2}=0.6$. The gravity darkening exponents were put to $g_{1}=g_{2}=0.32$ following Lucy (1967). We used an initial mass ratio of $q=0.725$ following Liu et al. (2012). The best model for V551 Aur consists of a detached configuration for the binary system with a mass ratio $q=0.769 \pm$ 0.005 . The temperature of secondary component, $T_{2}$, is about $5900 \mathrm{~K}$. Table 3 shows the final values for the system parameters including orbital inclination $(i)$, the dimensionless effective potentials of 


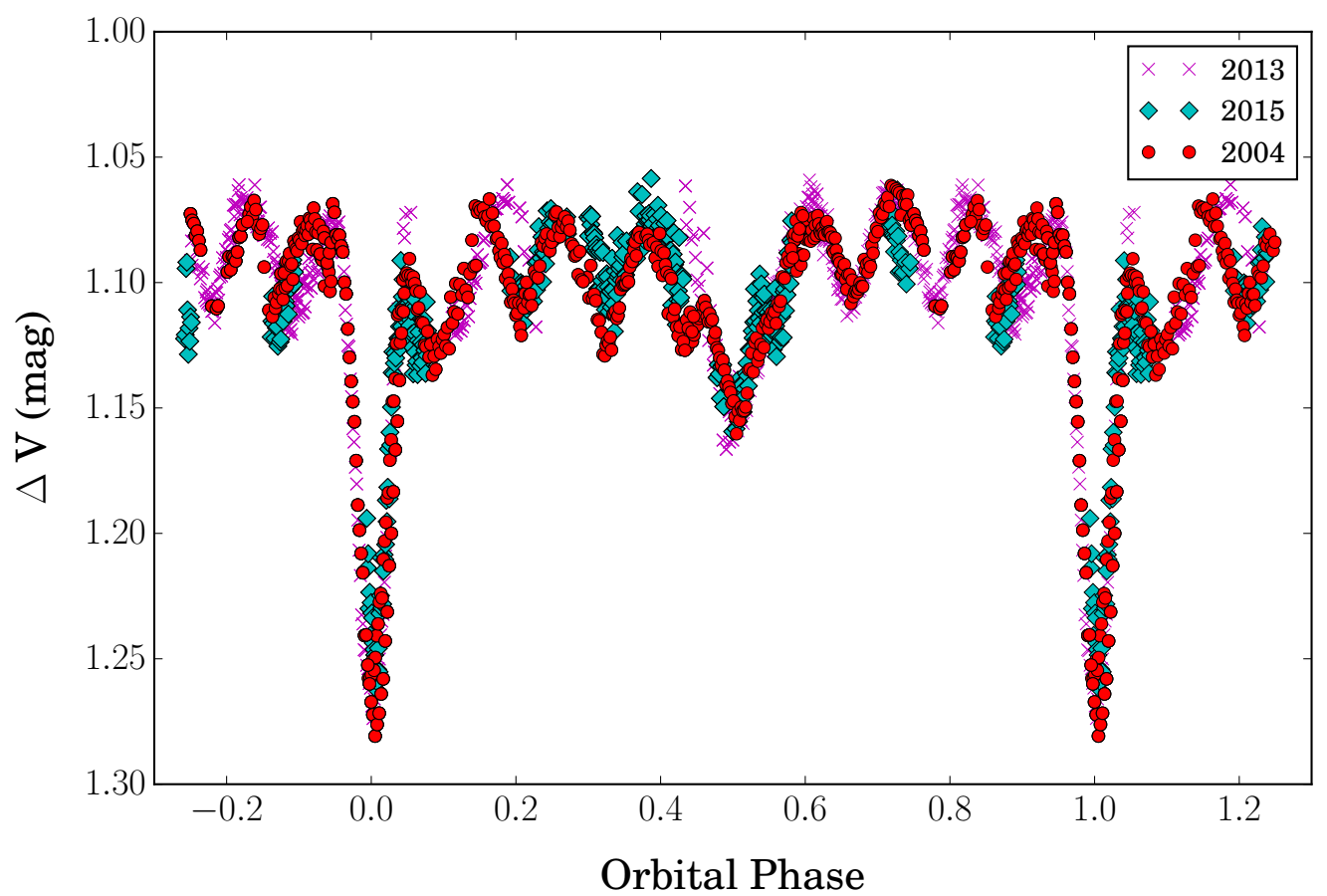

Figure 2: Differential $V$ observations of V551 Aur folded in the phase diagram according to the new ephemeris as given in Eq. (1).

the primary and secondary stars $\left(\Omega_{1,2}\right)$, the ratio between primary star and total luminosities $\left(L_{1} /\left(L_{1}+\right.\right.$ $\left.L_{2}\right)$ ), and the various radii characterizing the stellar shape for both stars.

\subsection{Period Analysis}

We calculated the residuals after subtraction of the binary model from the original light curve. Intervals with eclipse minima were excluded from these residuals for a consecutive period analyses with the program Period04 (Lenz \& Breger 2005). Only the DFT peaks with a signal-to-noise ratio (S/N) larger than 4.0 (Breger 1993) were considered as significant signals. These signals were removed from the data and the new residuals were analyzed. As a result, we detected two frequencies of $f_{1}=$ $7.713147(2) \mathrm{c} / \mathrm{d}$ (semi amplitude of $18.7(0.3) \mathrm{mmag}$ ) and $f_{2}=15.47419(1) \mathrm{c} / \mathrm{d} . f_{2}$ is identified as the first harmonic of $f_{1}$.

\subsection{Mode identifications}

We derived the $Q$-value of $Q_{1}=0.095 \pm 0.017$ days for $f_{1}$ by using the expression (Breger 1990):

$$
\log Q=-\log f+3.33 \log g+0.1 \log M_{b o l}+10 \log T_{\text {eff }}-6.456
$$

For the acoustic radial and non-radial modes of models of $\delta$ Scuti variables from 1.5 to 2.5 solar masses, the $Q$-values are essentially smaller than $\sim 0.033$ days (Fitch, 1981). Therefore, V551 Aur might be a g-mode pulsator. We found the accurate ratio of orbital to pulsation period to be $9.04878 \pm$ 0.00001 , which is within 5\% of an integer value. However, the degree $l$ and the azimuthal number $m$ of the pulsation modes are still unknown, so the pulsation period in the co-rotating frame of the pulsating 
Table 2: Times of light minima of V551 Aur originating from the literature and from this research. The residuals (O-C) were computed with the new ephemeris. Ref: (1) is Gaspar et al. (2003), (2) is Zhang et al. (2012), (3) is this research.

\begin{tabular}{cccccc}
\hline $\begin{array}{c}\text { Time of minimum } \\
(\text { HJD }+2450000)\end{array}$ & Epoch & $O-C$ & Min & Filter & Ref \\
\hline 2307.408 & -284.0 & -0.0134 & I & V & $(1)$ \\
2308.583 & -283.0 & -0.0121 & I & V & $(1)$ \\
2640.605 & 0.0 & 0.0 & I & V & $(1)$ \\
3088.757 & 382.0 & -0.0006 & I & V & $(3)$ \\
5562.970 & 2491.0 & -0.0098 & I & V & $(2)$ \\
5562.973 & 2491.0 & -0.0077 & I & B & $(2)$ \\
5565.316 & 2493.0 & -0.0108 & I & V & $(2)$ \\
5565.315 & 2493.0 & -0.0109 & I & B & $(2)$ \\
5567.086 & 2494.5 & -0.0018 & II & V & $(2)$ \\
5567.085 & 2494.5 & -0.0024 & II & B & $(2)$ \\
5568.259 & 2495.5 & -0.0018 & II & V & $(2)$ \\
5568.258 & 2495.5 & -0.0030 & II & B & $(2)$ \\
5570.009 & 2497.0 & -0.0099 & I & V & $(2)$ \\
5570.010 & 2497.0 & -0.0090 & I & B & $(2)$ \\
5571.183 & 2498.0 & -0.0092 & I & V & $(2)$ \\
5571.184 & 2498.0 & -0.0090 & I & B & $(2)$ \\
5572.359 & 2499.0 & -0.0068 & I & V & $(2)$ \\
5572.357 & 2499.0 & -0.0087 & I & B & $(2)$ \\
6330.233 & 3145.0 & -0.0045 & I & V & $(3)$ \\
6330.233 & 3145.0 & -0.0036 & I & B & $(3)$ \\
\hline
\end{tabular}

component cannot be determined yet. We therefore believe that g-mode pulsations in V551 Aur are in tidal resonance with the orbital period.

\section{Discussion and Conclusions}

The CCD times of the light mininma of V551 Aur in the $V$-band were obtained in the years 2004, 2013 and 2015 by using the $1-\mathrm{m}$ telescope at the LOAO, Arizona and the 0.5 and 2.4-m telescopes at TNO, Thailand. From this study, we obtained a new value of the orbital period of V551 Aur (1.1731744 \pm 0.0000002 days). The light curve was analyzed using the Wilson-Devinney technique in order to obtain a new set of systems parameters. According to our analysis, the solution shows that V551 Aur is a detached binary system with $q=0.769$ and $i=73.01$ degrees. From the period analysis of the residuals, we confirm the presence of pulsations with a main frequency of $7.71315 \mathrm{c} / \mathrm{d}$. The results show that $f_{2}$ is the first harmonic of $f_{1}$. The ratio between the orbital and pulsation period (9.049) hints towards the existence of a resonance or a possible causal relation to tidal effects suggesting that the 9th harmonic of the orbital period is exciting g-mode pulsations by the tidal forces. The presented results are part of a more extended photometric study of time series observations for the open cluster NGC 2126 with the purpose to detect new variable stars, to study the light curves of binary systems as well as the oscillation spectra of the pulsating stars. 
Table 3: System Parameters

\begin{tabular}{cc}
\hline Parameters & Best-fit value \\
\hline$P(\mathrm{~d})$ & $1.1731744 \pm 0.0000002$ \\
$i\left(\left(^{\circ}\right)\right.$ & $73.01 \pm 0.06$ \\
$q=m_{1} / m_{2}$ & $0.769 \pm 0.005$ \\
$T_{1}(\mathrm{~K})$ & 7000 (fixed) \\
$T_{2}(\mathrm{~K})$ & $5938 \pm 23$ \\
$\Omega_{1}$ & $5.38 \pm 0.03$ \\
$\Omega_{2}$ & $4.84 \pm 0.02$ \\
$L_{1} /\left(L_{1}+L_{2}\right)$ & $0.680 \pm 0.005$ \\
$r_{1, \text { back }}\left(\mathrm{R}_{\odot}\right)$ & $0.220 \pm 0.002$ \\
$r_{1, \text { side }}\left(\mathrm{R}_{\odot}\right)$ & $0.218 \pm 0.002$ \\
$r_{1, \text { pole }}\left(\mathrm{R}_{\odot}\right)$ & $0.216 \pm 0.002$ \\
$r_{1, \text { point }}\left(\mathrm{R}_{\odot}\right)$ & $0.221 \pm 0.002$ \\
$r_{2, \text { back }}\left(\mathrm{R}_{\odot}\right)$ & $0.211 \pm 0.002$ \\
$r_{2, \text { side }}\left(\mathrm{R}_{\odot}\right)$ & $0.208 \pm 0.002$ \\
$r_{2, \text { pole }}\left(\mathrm{R}_{\odot}\right)$ & $0.205 \pm 0.002$ \\
$r_{2, \text { point }}\left(\mathrm{R}_{\odot}\right)$ & $0.012 \pm 0.002$ \\
\hline
\end{tabular}

\section{Acknowledgements}

This research is part of $\mathrm{PhD}$ Thesis of $\mathrm{N}$. Chehlaeh at Chiang Mai University under the supervision of D. Mkrtichian, P. Lampens and S. Komonjinda. This study is supported by the Development and Promotion of Science and Technology Talents Project (DPST). The authors would like to thank the Royal Observatory of Belgium and the National Astronomical Research Institute of Thailand for their support in this work. A. Kusakin acknowledges his work as a research activity in the framework of Project No. 0075/GF4 "Investigation of the variability of stars and the processes of substance exchange with a gas-dust disk". The search has made use of the WEBDA database. The following Internet-based resources were used in research for this paper: the NASA Astrophysics Data System; the SIMBAD database and the ViziR service operated by CDS, Strasbourg, France; the arXiv scientific paper preprint service operated by Cornell University.

\section{References}

Breger M. 1990, DSSN, 2, 13

Breger M., Stich J., Garrido R. et al. 1993, A\&A, 271, 482

Cuffey J. 1943, ApJ, 97, 93

Fitch W. S. 1981, ApJ, 249, 218

Frandsen S., Arentoft T. 1998, JAD, 4

Gaspar A., Kiss L. L., Bedding T. R. et al. 2003, A\&A, 410, 879

Glushkova E. V., Zabolotskikh M. V., Koposov S. E. et al. 2013, MNRAS, 429, 1102-1112

Han W., Mack P., Lee C. U. et al. 2005, PASJ, 57, 821

Lenz P., Breger M. 2005, CoAst, 146, 53

Liu N., Zhang X.B., Ren A. B., Deng L. C., Luo Z. Q. 2012, A\&A, 12, 671

Liu S. F., Wu Z. Y., Zhang X. B. et al. 2009, A\&A, 9, 791

Lucy L. B. 1967, ZAp, 65, 89

Lynga G. 1995, yCat, 7092

Mkrtichian D. E., Kusakin A. V., Gamarova A. Y., Nazarenko V. 2002, ASPL, 96

Prša A., Zwitter T. 2005, ApJ, 628, 426 
Tapia M. T., Schuster W. J., Michel R. et al. 2010, MNRAS, 401, 621 van Hamme W. 1993, AJ, 106, 2096

Wilson R. E., Devinney E. J. 1971, ApJ, 166, 605

Zacharias N., Finch C. T., Girard T. M. et al. 2013, AJ, 145, 44

Zhang X. B., Deng L. C., Luo C. Q. 2012, AJ, 144, 141

Zhang X. B., Deng L. C., Tian J. F. et al. 2014, AJ, 148, 106 